\title{
PECULIARITIES OF THE SCHLIEREN TEXTURES IN LYOTROPIC NEMATIC MESOPHASES
}

\author{
A. Nesrullajev and N. Kazanci \\ Department of Physics, Faculty of Science, Ege University \\ 35100 Bornova-İzmir, Turkey \\ (Received December 17, 1998)
}

\begin{abstract}
In the present paper the peculiarities of schlieren textures of lyotropic nematic mesophases have been investigated by polythermic polarization microscopy and capillary temperature wedge methods. The lyotropic nematic-calamitics $\left(N_{c}\right)$ and lyotropic nematic-discotics $\left(N_{\mathrm{d}}\right)$ formed in binary and ternary systems on the base of tetradecyldimethyl amino bromide and tetradecyldimethyl amino oxide have been the subjects of our investigations. The dynamics of time and temperature transformations of schlieren textures have been studied. The comparative estimation of schlieren textures of $N_{\mathrm{c}}$ and $N_{\mathrm{d}}$ mesophases has been performed. The optical force and optical sign of the schlieren textures observed have been determined.
\end{abstract}

PACS numbers: 42.70.Df, 82.70.-y, 82.70.Dd

\section{Introduction}

One of the main peculiarities of liquid crystals is their high sensitivity to different external effects. In particular, hydrodynamic flows, deformations, different surfaces affect the micro- and macroscopic properties of liquid crystals. The variation of the above properties has an effect on the character of the liquid crystal texture. Among these textures, of great interest are the textures attributed to the disturbance of optical continuity of liquid-crystalline mesophase. The optical continuity disturbances, i.e. the breaking of the field of the vector-director $\boldsymbol{n}$, are due to different types of defects. In liquid crystals the defects often appear as a result of inhomogeneity of supporting surfaces, effects of mechanical inclusions and mechanical deformations. The above defects can also occur during the melting process of mesogene from the crystalline state to the mesophase, at the applied electric and magnetic fields, and with the temperature gradients. In this case, the defects in liquid crystals differ from those in solid crystals by the fact that they can be observed and their peculiarities can be investigated by polarization-microscopy technique.

In the present paper the configuration of textures with different optical sign and different disclinations of strength has been studied by polythermic polarization microscopy and capillary temperature wedge methods and using the optical mapping technique. 


\section{Samples, methods and theory}

\subsection{Samples}

In the present paper, the subjects of our investigations were the lyotropic mesophases of nematic-calamitic $\left(N_{\mathrm{c}}\right)$ and nematic-discotic $\left(N_{\mathrm{d}}\right)$. The structural units of $N_{\mathrm{c}}$ and $N_{\mathrm{d}}$ mesophases are rod-like (Fig. 1a) and disk-like (Fig. 1b) micelles, respectively. Both nematic mesophases are uniaxial and are characterized by the long-range orientation order. The investigation carried out in $N_{\mathrm{c}}$ and $N_{\mathrm{d}}$ mesophases oriented by shift deformation have shown that the uniaxial mesophases $N_{c}$ and $N_{d}$ are, respectively, optically positive and negative. This is attributed to the fact that the amphiphile molecules in micelles of the mesophase $N_{\mathrm{c}}$ are located perpendicularly to the vector-director coinciding with the direction of the long axes of rod-like micelles. However, in micelles of the mesophase $N_{\mathrm{d}}$ the amphiphile molecules are located parallel to the vector-director which is perpendicular to the plane of disk-like micelles.

(a)

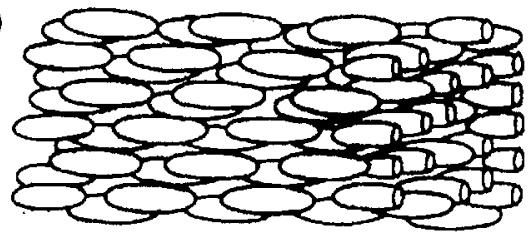

(b)

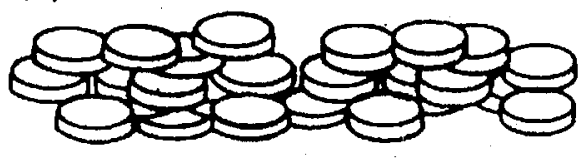

Fig. 1. Schematical appearance of nematic-calamitic (a) and nematic-discotic (b) mesophases.

The mesophase $N_{\mathrm{c}}$ was obtained in binary tetradecyldimethyl amino bromide (TDDABr) +water and tetradecyldimethyl amino oxide (TDDAO)+water systems, while the mesophase $N_{\mathrm{d}}$ was obtained in ternary $\mathrm{TDDABr}+$ water + decanol and TDDAO + water + decanol systems. The phase diagrams of $\mathrm{ly}$, ropic systems based on TDDABr and TDDAO are shown in Refs. [1,2]. The amphiphile substance and decanol were obtained from Merck AG. The water used as solvent was twice distilled with a simultaneous deionization.

The samples studied are the plane capillaries in the form of sandwich-cell. The layer thickness of the liquid crystals was prescribed by the mylar films and was $20 \mu \mathrm{m}$.

\subsection{Investigation methods}

The investigations of morphological properties and peculiarities of schlieren textures of $N_{\mathrm{c}}$ and $N_{\mathrm{d}}$ mesophases were carried out by polythermic polarization microscopy and methods of the crystallophysics using the Olympus BX-P polarization microscope and a microphotographical system. A disclination of strength and the optical sign of disclinations round the singular points were studied by means of optical mapping technique [3, 4]. Investigation of the temperature variations of lyotropic nematic mesophase textures in the lyotropic nematic $\rightarrow$ isotropic 
liquid phase transition region and determination of the phase transition temperatures were made using the capillary temperature wedge described previously in Refs. $[5,6]$.

\subsection{Theory}

In liquid crystals the defects or disclinations, as they were called by Frank [7], can be divided into three types: the point disclinations, the linear disclinations and two-dimensional disclinations or the walls $[3,8]$. The point disclinations of molecular sizes are attributed to the rotation of liquid crystal molecules along the long and short axes with a precession and deviation of the long axes of molecules from the vector-director direction by the magnitude of about one degree. The above disclinations are not revealed in the textures. The linear disclinations are the most common types of defects in liquid crystals determining the type of liquid crystal textures. Neddle-type nematic textures and the schlieren textures of nematics and smectics refer to this type of textures. Two-dimensional disclinations, i.e. confocal and polygonal formations in polycrystalline textures, also define the type of the observed textures.

A general theory of the disclinations was considered in Refs. [9, 10]. Different aspects of disclinations and defect problems in liquid crystals were discussed in Refs. [11-16].

The schlieren textures of lyotropic nematics were the subject of our investigations. As in the schlieren textures the wedge-shaped disclinations are the most prominent, we consider briefly a theory of the wedge-shaped disclinations.

Assume that the vector-director $n$ of the nematic lies in the $x y$ plane, while a wedge-shaped disclination lies along the $z$-axis. Then, the expression for the density of nematic free energy

$$
\mathcal{J}=\int_{V} F \mathrm{~d} V
$$

can be written as [17]

$$
\mathcal{J}=\frac{1}{2} \int_{V}\left[k_{11}(\operatorname{div} n)^{2}+k_{33}(n x \operatorname{rot} n)^{2}\right] \mathrm{d} V,
$$

where $k_{11}$ and $k_{33}$ are the elastic constants of transverse and longitudinal bending, respectively. Taking into account that the vector-director projections on the $x, y$ and $z$ axes have the form

$$
n_{x}=\cos \theta, \quad n_{y}=\sin \theta, \quad n_{z}=0,
$$

where $\theta$ is the tilt angle of the vector-director to the $x$-axis, in a single-constant-approximation to the expression for the free energy density will be

$$
\mathcal{J}=\frac{1}{2} k \int_{V}\left[\left(\frac{\partial \theta}{\partial x}\right)^{2}+\left(\frac{\partial \theta}{\partial y}\right)^{2}\right] \mathrm{d} V .
$$

Minimizing this functional by the solution of the Laplace equation, we obtain the equilibrium condition formulated first by Frank [7]

$$
\nabla^{2} \theta=0
$$


Transforming to a cylindrical coordinate system, converting the Cartesian coordinates $x y z$ to the cylindrical coordinate system $r d z$, taking into account that, as it was mentioned above, the $z$-axis of spatially fixed system $x y z$ is directed perpendicularly to the nematic layer

$$
x=r \cos \alpha, \quad y=r \sin \alpha, \quad z=z .
$$

Then, Eq. (5) has the form

$$
\frac{1}{r^{3}} \frac{\partial^{2} \theta}{\partial \alpha^{2}}=0
$$

The following expression is the solution of Eq. (7) [13]:

$$
\psi=S \alpha+\theta_{0}
$$

Here $\theta_{0}$ is the initial angle of rotation of the vector-director, $S$ is a disclination of strength. The constant $S$ is obtained from the condition of unambiguity of the solution, namely, at the variation of the rotation $\psi$ of the vector-director $n$ by $\pi$, the same vector-director $n$ should be obtained. As the nematic mesophase is unpolar, then $n=-n$. Such a variation of rotation of vector-director will correspond to the variation of the polar angle $\alpha$ by $2 \pi$.

Theoretically, for the nematics with a. point symmetry one can obtain disclinations with any strength: $S= \pm \frac{1}{2}, \pm 1, \pm \frac{3}{2}, \ldots$ However, only the disclinations with the strength: $S=-\frac{1}{2},+\frac{1}{2},-1,+1$, were observed experimentally. The disclinations of high strength (disclinations with great Frank indices) are of high energy as they are proportional to the square of the Frank index. Therefore, the high energy disclinations are unstable [7].

\section{Results and discussion}

As soon as the plane capillary was filled up with the liquid crystal, an unstable weak birefringent texture was observed both for $N_{\mathrm{c}}$ and $N_{\mathrm{d}}$ mesophases. For 30-60 minutes the gradual transformations of textures were observed. In this case, a birefringence of certain formations and the sizes of birefringent regions increased. As a result, a stable texture not changing for a long time occurred in hermetic samples. The occurrence of such a texture was attributed to the micellar interactions and the interactions between micelles and orienting surfaces of the plane capillaries. Due to a rather high viscosity of $N_{\mathrm{c}}$ and $N_{\mathrm{d}}$ mesophases, a stable texture has appeared for a rather long period of time.

The schlieren textures of nematic mesophases observed at room temperature in lyotropic systems investigated are shown in Figs. 2 and 3. In the above figures one can observe distinctly the systems of points and the beams of dark bands emerging from these points. As seen in Figs. 2 and 3, one band of each pair is more distinct than the other one which has a diffuse character. These bands are attributed to linear disclinations which are perpendicular to a liquid crystal layer. In such bands a local optical axis of a liquid crystal was parallel or perpendicular to the polarization plane of the incident light and therefore, the extinction was observed when using the crossed polarizers.

It is seen in Figs. 2 and 3 that two or four dark bands are emerging from singular points of observed schlieren textures. This indicates the disclinations 

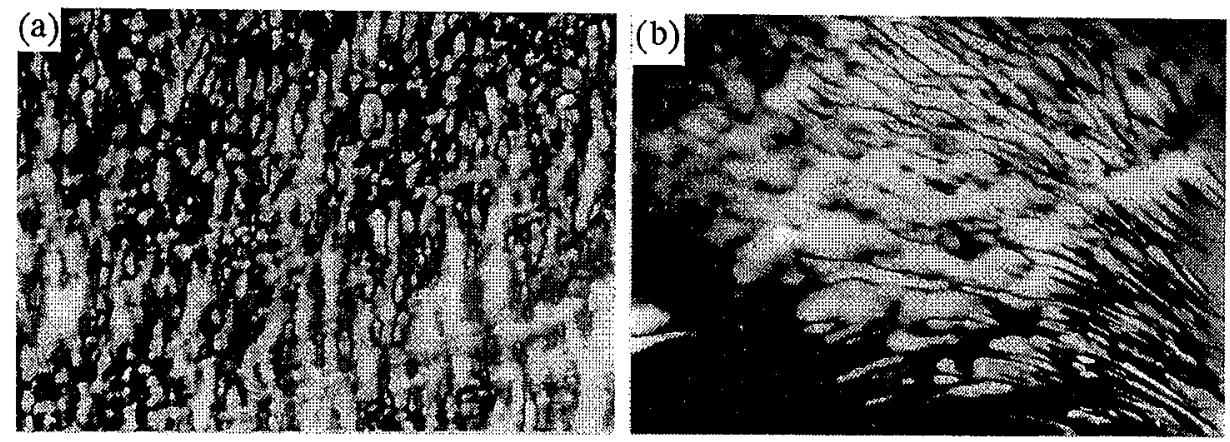

Fig. 2. Schlieren textures of lyotropic nematic mesophases based on tetradecyldimethyl amino oxide. Magnification $\times 100$, temperature $295 \mathrm{~K}$, crossed polarizers; (a) nematic-calamitic mesophase; (b) nematic-discotic mesophase.
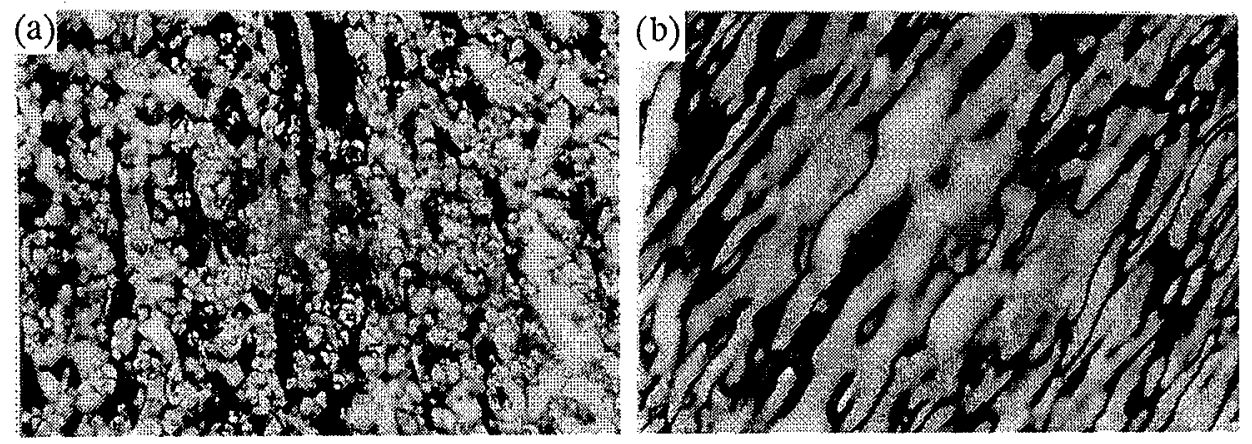

Fig. 3. Schlieren textures of lyotropic nematic mesophases based on tetradecyldimethyl amino bromide. Magnification $\times 100$, temperature $295 \mathrm{~K}$, crossed polarizers; (a) nematic-calamitic mesophase; (b) nematic-discotic mesophase.

strengths $S=\frac{1}{2}$ and $S=1$, respectively. The disclination sign was determined by optical mapping technique $[3,4]$ consisting in a comparative investigation of band variation at the rotation of polarizers in a crossed position relative to the fixed sample. Thus, at the rotation of the crossed polarizers the dark bands gradually disappear indicating a gradual change of vector-director around the disclinations. For one and the same sample the direction of rotation of the dark bands emerging from some singular points coincided with the direction of the crossed polarizers rotation (positive disclinations), while for the other bands emerging from other singular points the direction of rotation of bands was opposite to the direction of crossed polarizers rotation (negative disclinations).

As seen from the comparison of Figs. 2 and 3, the schlieren textures of $N_{\mathrm{c}}$ and $N_{\mathrm{d}}$ mesophases are different. The peculiarity of schlieren textures in $N_{\mathrm{d}}$ mesophase is the existence of inversion walls. These walls are nearly parallel dark (or light versus the position of the crossed polarizers) lines comnecting the wedge-shaped disclinations $[12,18]$. The above walls were perpendicular to the liquid crystal layer and were the transition region between the planar orientation regions. On 
the inversion walls the molecules changed their orientation by $\pi$. However, as the molecular orientation was changed by $\pi$ at the intersection of the wall (Figs. 2 and 3), the strength of singularities localized in the inversion wall was $S= \pm 1$. In this case, as a rule, in the center of the region limited by the dark bands the birefringence value was considerably higher. Note that some inversion walls are characterized by disclinations within themselves (Figs. 2a and 3a).

The peculiarity of schlieren textures of $N_{c}$ mesophase is the existence of unstretched regions with a high birefringence and limited by the dark closed lines (Figs. $2 \mathrm{~b}$ and $3 \mathrm{~b}$ ). These closed lines are specific inversion walls. It should be noted that some of them emerge from disclinations with $S= \pm 1$. The optical investigations have shown that in most cases the above disclinations are the surface disclinations. The inversion walls stretched from one supporting surface of the plane capillary to another in this case. The point defects are also seen in Figs. 2b and $3 \mathrm{~b}$. The optical strength of the observed point defects was $S= \pm 1$. In this case, the estimations have shown that the tilt angle of micelles to the surface can be positive and negative. The shift of supporting surfaces of plane capillaries leads to disappearance of the point defects indicating their relation to supporting surfaces.

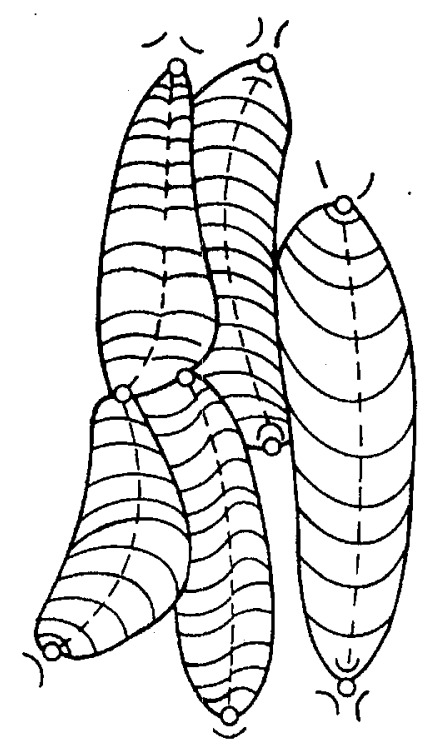

Fig. 4. Scheme of molecular alignment in inversion walls.

The orientations of the vector-lines constructed in accordance with Figs. 2 and 3 are schematically shown in Fig. 4 . The heavy lines denote the inversion wall boundaries. A general variation of micellar orientation at the intersection of wall is equal to $\pi$. The variation by $\pi / 2$ was achieved in the wall center. This is shown by the dashed line. In some singular points a distinct and diffuse bands changed their position relative to a central line. The intersection points in this case are the singularities with $S=-1$ and $S=+1$. 


\section{Acknowledgment}

This work has been supported by the Science Foundation of Ege University; grant No. $98 /$ Fen/002.

\section{References}

[1] G. Hertel, H. Hoffmann, G. Oetter, B. Schwandner, Progr. Colloid Polym. Sci. 73, 95 (1987).

[2] G. Bartusch, H.D. Dörfler, H. Hoffmann, Progr. Colloid Polym. Sci. 89, 307 (1992).

[3] J.E. Zimmer, J.L. White, Mol. Cryst. Liq. Cryst. 38, 177 (1977).

[4] J.E. Zimmer, PhD Thesis, Pardue University, W. Lafayette, Indiana, 1978.

[5] A. Nesrullajev, S. Salihoğlu, H. Yurtseven, Int. J. Mod. Phys. B 12, 213 (1998).

[6] A. Nesrullajev, A. Bahçe, Tr. J. Phys. 21, 822 (1997).

[7] F.C. Frank, Disc. Faraday Soc. 25, 1 (1958).

[8] W.F. Harris, Surf. Defect. Prop. Solids 3, 57 (1974).

[9] F.R.N. Nabarro, Theory of Crystal Dislocations, Clarendon Press, Oxford 1967.

[10] E. Kröner, K.H. Anthony, Ann. Rev. Mater. Sci. 5, 43 (1975).

[11] P.G. de Gennes, Physics of Liquid Crystals, Clarendon Press, Oxford 1974.

[12] A. Saupe, Mol. Cryst. Liq. Cryst. 21, 211 (1973).

[13] S. Chandrasekhar, Liquid Crystals, Cambridge Univ. Press, Cambridge 1977.

[14] M.J. Stephen, J.P. Straley, Rev. Mod. Phys. 46, 617 (1974).

[15] M. Kleman, in: Adv. Liq. Cryst., Ed. G. Brown, Vol. 1, Academic Press, New York 1975 , p. 267.

[16] M. Kleman, in: Dislocations in Solids, Ed. F.R.N. Nabarro, Vol. 5, North Holland Publ., Amsterdam 1980, p. 243.

[17] A.S. Sonin, Introduction to the Physics of Liquid Crystals, Science Publ., Moscow 1983.

[18] J. Nehring, A. Saupe, J. Chem. Soc. Faraday Trans. 2, 68 (1972). 